and prostaglandin metabolism in zinc deficient pregnant rats. Life Sci 32:2471-2478

28. Everett $G$, Apger $J 1983$ Effect of zinc deficiency on prostaglandin $F_{2 \alpha}$ and $E$ in the rat. In: Bratter P, Schramel P (eds). Trace Elements-Analytic Chem- istry in Medicine and Biochemistry, Vol 2. Walter de Gruyter, NY, pp 219-226

29. Meydani SN, Dupont J 1982 Effect of zinc deficiency on prostaglandin synthesis in different organs of rat. J Nutr 6:1098-1104

0031-3998/85/1907-0700\$02.00/0

PEDIATRIC RESEARCH

Copyright (C) 1985 International Pediatric Research Foundation, Inc.

Vol. 19, No. 7, 1985 Printed in U.S.A.

\title{
Transfer and Metabolism of Carnitine and Carnitine Esters in the in Vitro Perfused Human Placenta
}

\author{
EBERHARD SCHMIDT-SOMMERFELD, DUNA PENN, RAVI J. SODHA, MARIETTE PRÖGLER, \\ MILAN NOVAK, AND HENNING SCHNEIDER \\ Department of Pediatrics, University of Giessen, Federal Republic of Germany; Division of Perinatal Physiology, \\ Department of Obstetrics, University of Zürich, Switzerland; and Department of Pediatrics, University of \\ Miami, Florida
}

\begin{abstract}
The transfer and metabolism of L-carnitine, $\mathrm{L}$-acetylcarnitine, and L-palmitoylcarnitine were studied in the human placenta at term by means of in vitro dual perfusion of a placental lobe. $\mathrm{C}$-Carnitine transfer was $20 \%$ that of the freely diffusing antipyrine and $40 \%$ that of $\mathrm{L}$ lysine. The transfer of $L$-acetylcarnitine was similar to that of L-carnitine, but no placental transfer of L-palmitoylcarnitine was found. In contrast to L-lysine, L-carnitine, and L-acetylcarnitine were not actively transported from the maternal to the fetal circulation. No stereospecific transfer of carnitine across the placenta was found. However, there was stereospecific uptake of carnitine by placental tissue. The placenta exhibited an active carnitine metabolism by esterifying free carnitine and hydrolyzing carnitine esters taken up from the perfusion medium and releasing the metabolites into the fetal and maternal circulations. (Pediatr Res 19: 700-706, 1985)
\end{abstract}

Carnitine plays an important role in fat catabolism by facilitating the transport of fatty acids across the mitochondrial membrane (1). Its availability seems to be essential during the immediate postnatal period when fat constitutes the major source of energy $(2,3)$. Decreased carnitine blood (4-6), tissue (7), and urine (5) concentrations in premature infants receiving carnitinefree parenteral nutrition provide indirect evidence that the fetus may not be capable of substantial carnitine synthesis. This is also suggested by the low activity of the key enzyme for carnitine biosynthesis during early human development (8).

Little is known about carnitine transfer across the placenta. Large species differences have been reported. Whereas an ex-

Received April 26, 1984; accepted March 6, 1985.

Reprint requests E. Schmidt-Sommerfeld, M.D., Zentrum für Kinderheilkunde, Justus-Liebig-Universität, Feulgenstr. 12, 6300 Giessen, F.R.G.

Supported by funds from the Deutsche Forschungsgemeinschaft and the Schweizer Nationalfond.

This paper is dedicated to Professor Helmut Wolf on the occasion of his 60th birthday. tremely slow carnitine placental transport has been found in the sheep in vivo, rapid transfer occurs across the guinea pig placenta (9). Until now, only indirect evidence exists for carnitine placental transfer in the human. A strong correlation has been found between maternal and umbilical carnitine blood concentrations (10). Nothing is known about the mechanism by which carnitine may cross the placenta. Free carnitine concentrations have been reported to be higher in umbilical than in maternal venous blood $(11,10)$. This finding has given rise to the speculation that active transport from the mother to the fetus occurs. However, we found decreased free carnitine and increased acylcarnitine concentrations in maternal blood at delivery compared with those from pregnant women not in labor (10). This could be attributed to acute changes in maternal fat metabolism and makes it difficult to draw conclusions about the role of the placenta in carnitine transfer from a comparison of maternal and umbilical blood levels. Moreover, carnitine acetyltransferase $(12,13)$ and carnitine palmitoyltransferase activities $(12,14)$ have been demonstrated in the human placenta suggesting that the placenta itself may actively participate in perinatal carnitine metabolism. To study human placental carnitine transfer and metabolism isolated from maternal or fetal metabolic influences, we performed experiments using the in vitro dual perfused human placental lobe.

\section{MATERIALS AND METHODS}

Perfusion technique. Twenty-seven placentas were obtained from uncomplicated term gestations by vaginal or abdominal deliveries and immediately prepared for study. Using the technique of dual perfusion of an isolated human placental lobe described by Schneider et al. (15), fine steel cannulas were inserted into the artery and vein supplying the chorionic vascular system. The lobe (mean weight $34.8 \mathrm{~g}$ ) was immediately perfused with fetal perfusate, removed from the rest of the placenta, and fixed with the maternal side upward in a thermoregulated plexiglass chamber. For establishment of the maternal circulation, three to five steel cannulas were inserted into the intervillous space by penetrating the decidual plate over the blanched area 
(maternal artery). Perfusate leaving the intervillous space through venous openings in the decidual plate was collected from the chamber by suction (maternal vein). The maximal time span between delivery of the placenta and establishment of both perfusion circuits was $30 \mathrm{~min}$.

The perfusates consisted of Earl's buffered salt solution containing human serum albumin $(0.4 \mathrm{~g} / \mathrm{dl})$ and glucose $(8.3 \mathrm{mM}$ on the maternal side; $2.8 \mathrm{mM}$ on the fetal side). They were kept in a water bath at $37^{\circ} \mathrm{C}$ and gassed with $95 \% \mathrm{O}_{2}$ and $5 \% \mathrm{CO}_{2}$. Maternal and fetal venous samples were collected at appropriate time intervals for analysis. Flow and pressure were continuously monitored in both circuits. Flow rates ranged from $9-15 \mathrm{ml} /$ min on the maternal and from $7-14 \mathrm{ml} / \mathrm{min}$ on the fetal side, depending on the size of the perfused placental lobe.

Perfusion pressures ranged from $35-55 \mathrm{~mm} \mathrm{Hg}$ in the fetal circulation. To judge the viability of the preparation, glucose consumption and lactate and pyruvate production were measured.

In five cases, the experiments had to be interrupted for technical reasons, e.g. major leaks, and were excluded from evaluation.

Experimental Design. Series I. In five experiments both circulations were kept open, i.e. the perfusates were not recirculated. After 12 min of perfusion with carnitine free medium, L-carnitine (30 $\mu \mathrm{M}$, Serva Chemical Co.), L-acetylcarnitine ( $30 \mu \mathrm{M}$, Serva Co.), and L-palmitoylcarnitine ( $20 \mu \mathrm{M}$, Serva Co.) were sequentially added in three experimental phases (duration $30 \mathrm{~min}$ ) to the maternal perfusate which contained antipyrine $(0.42 \mathrm{mM})$ as reference substance. The sequence of addition was alternated among experiments. There was no carnitine or antipyrine in the fetal perfusate. The placental lobe was perfused with carnitinefree medium for $15 \mathrm{~min}$ between the three phases. Maternal and fetal venous samples were collected after 10,20 , and $30 \mathrm{~min}$ of perfusion in each phase. One sample pair was obtained for carnitine measurement at the end of each carnitine-free period to measure carnitine release from the placenta and to assure complete washout of carnitine from the intravascular space.

Transfer and clearance of carnitine, carnitine esters, glucose, and antipyrine were calculated from the mean of three measurements according to the following formulas (16):

$$
\begin{aligned}
\text { Transfer } & =\frac{\left(C_{F V}-C_{F A}\right) \cdot Q_{F}}{\text { weight of perfused placental lobe }} \text { and } \\
\text { Clearance } & =\frac{\left(C_{F V}-C_{F A}\right) \cdot Q_{F}}{C_{M A}-C_{F A}}
\end{aligned}
$$

where $\mathrm{C}$ is the concentration in the maternal artery (MA) and vein (MV) and fetal artery (FA) and vein (FV). $Q_{F}$ refers to the fetal flow rate. Transfer and clearance did not systematically vary during the course of perfusion. To reduce the influence of hemodynamic, anatomic, and technical variables among the experiments (17), the clearance was also expressed as percentage of antipyrine clearance (clearance index).

Series II. In nine experiments, both the maternal and fetal perfusates contained equal concentrations of ${ }^{3} \mathrm{H}$-L-lysine $(0.14$ $\mathrm{mM} ; 1.5 \mu \mathrm{Ci} /$ liter, New England Nuclear) and L-carnitine (30 $\mu \mathrm{M})$ or L-acetylcarnitine $(30 \mu \mathrm{M})$. Antipyrine was present only in the maternal perfusate. After $25 \mathrm{~min}$ with both circulations open, the fetal circulation was closed, i.e. the fetal perfusate (approximately $100 \mathrm{ml}$ ) was recirculated. The maternal circulation remained open throughout the experiments so that the concentrations of all substances offered to the placenta were constant. After $30,60,90,120$, and 150 min of fetal recirculation, samples were collected from both circulations.

Series III. In eight experiments, the following substances were added to the maternal perfusate: 1$){ }^{14} \mathrm{C}$-L-carnitine $(30 \mu \mathrm{M} ; 2.5$ $\mu \mathrm{Ci} /$ liter) and ${ }^{3} \mathrm{H}$-L-lysine $0.14 \mathrm{mM} ; 20 \mu \mathrm{Ci} /$ liter $), n=4$; and 2 ) ${ }^{14} \mathrm{C}$-L-carnitine (30 $\mu \mathrm{M} ; 2.5 \mu \mathrm{Ci} /$ liter, Amersham) and ${ }^{3} \mathrm{H}-\mathrm{D}$ carnitine (30 $\mu \mathrm{M} ; 10 \mu \mathrm{Ci} /$ liter, Amersham), $n=4$.
The fetal perfusate contained the unlabeled substances in the same concentrations. As in series II, the maternal circulation was kept open, the fetal perfusate was recirculated, and samples were taken at the same intervals. At the end of the experiment the fetal perfusate was collected for chromatographic analysis. Thereafter, the perfusion was continued for $10 \mathrm{~min}$ with unlabeled medium to remove as much radioactivity as possible from the intravascular space. The perfused placenta was then frozen in liquid nitrogen.

Differentially labeled L- and D-isomers were compared in order to detect stereospecific transport mechanisms for the uptake of carnitine. Isotope uptake from the maternal perfusate is accounted for by 1) retention in the placental tissue and 2) transfer into the fetal circulation which we called transplacental exchange since there was equal substrate concentration on both sides of the placental barrier.

Uptake, placental retention, and transplacental exchange are expressed in the following relative terms (18):

Relative placental uptake (L:D)

$$
\begin{aligned}
& \qquad=\frac{M A_{L}-M V_{L}}{M A_{L}} \cdot \frac{M A_{D}}{M A_{D}-M V_{D}} ; \\
& \text { Relative transplacental exchange (L:D) }=\frac{F V_{L}}{M A_{L}} \cdot \frac{M A_{D}}{F V_{D}} ; \text { and } \\
& \text { Relative placental retention (L:D) }=\frac{P_{L}}{M A_{L}} \cdot \frac{M A_{D}}{P_{D}}
\end{aligned}
$$

where MA, MV, FV, and $P$ are the corresponding levels of radioactivity in the maternal artery, maternal vein, fetal vein, and placenta. $L$ and $D$ refer to the $L-$ and $D$-isomers of carnitine.

Analytical methods. The perfusion media were immediately centrifuged to remove red blood cells and the supernatants stored frozen at $-32^{\circ} \mathrm{C}$ until analysis. Free and total carnitine were measured by a modification (10) of the enzymatic radiochemical method of McGarry and Foster (19). Long-chain acylcarnitine was measured by the method of Brass and Hoppel (20). Glucose (21), pyruvate (22), lactate (23), and antipyrine (24) were measured with an autoanalyzer. The radioactivity was measured in two channels $\left({ }^{3} \mathrm{H} /{ }^{14} \mathrm{C}\right)$ with a Phillips liquid scintillation spectrometer.

For chromatographic separation of ${ }^{14} \mathrm{C}$-labeled carnitine derivatives, $10 \mathrm{ml}$ perfusate were mixed with $1 \mathrm{ml}$ perchloric acid $(2.5$ $\mathrm{M})$ and centrifuged. The precipitate was analyzed for radioactivity. The supernatant was adjusted to $\mathrm{pH} 6.5$ with $\mathrm{KOH}$ and freeze-dried. The resulting powder was dissolved in $0.5 \mathrm{ml}$ methanol/water $(9: 1, v / v)$, put on a silica gel column (Kieselgel $60 \mathrm{H}$, Merck Co., $16 \times 10 \mathrm{~mm}$ ), and eluted with acidified methanol/ water $(9: 1, \mathrm{v} / \mathrm{v}, \mathrm{pH} 5-6)$. The first $4 \mathrm{ml}$ of eluate containing salts, but no radioactivity, were discarded. The following $12 \mathrm{ml}$ of eluate containing almost all the radioactivity were collected and evaporated under a stream of nitrogen. After addition of $D, L-$ carnitine- $\mathrm{HCl}(0.25 \mu \mathrm{mol})$ and L-acetylcarnitine- $\mathrm{HCl}(0.20$ $\mu \mathrm{mol})$ as internal standards, the sample was dissolved in a small volume of methanol, spotted on a precoated thin-layer chromatography plate (Kieselgel 60, Merck $\mathrm{Co}$.) and developed in a system containing chloroform/methanol/water/conc. ammonia/ conc. formic acid $(55: 35: 5: 2: 3, \mathrm{v} / \mathrm{v})$. The spots were visualized in iodine vapors and marked. The $R_{f}$ values were 0.23 for free carnitine and 0.26 for acetylcarnitine. After evaporation of the iodine, the spots were scraped directly into scintillation vials and counted. The area between the acetylcarnitine spot and the solvent front which presumably contained other short- and medium-chain acylcarnitines was also scraped from the plate and measured for radioactivity. The concentrations of ${ }^{14} \mathrm{C}$-labeled carnitines were expressed as percentage of total radioactivity recovered from the plate.

Unperfused placental tissue was measured for total acid soluble and free carnitine as previously described (7). Placental tissue 
perfused with radiolabeled carnitine was homogenized in ice cold perchloric acid $(0.6 \mathrm{M})$, centrifuged, and the supernatant was analyzed for labeled carnitine and carnitine esters as described above. The precipitate was washed three times with perchloric acid, hydrolyzed with $\mathrm{KOH}$, reprecipitated with perchloric acid, and the resulting supernatant was measured for radioactivity of long-chain acylcarnitines.

\section{RESULTS}

Viability parameters. Glucose consumption was $0.16 \pm 0.11$ $\mu \mathrm{mol} / \mathrm{min} \cdot \mathrm{g}$; lactate production was $0.39 \pm 0.15 \mu \mathrm{mol} / \mathrm{min} \cdot \mathrm{g}$; and pyruvate production was $0.02 \pm 0.01 \mu \mathrm{mol} / \mathrm{min} \cdot \mathrm{g}$ perfused tissue (means $\pm \mathrm{SD}$ ). These parameters did not vary significantly throughout the duration of perfusion (approximately $150 \mathrm{~min}$ ).

Series I. During the initial perfusion with carnitine-free medium small concentrations of free and acylcarnitine were measured in the maternal ( $1.4 \pm 0.4$ and $0.9 \pm 0.8 \mu \mathrm{M}$, respectively) and the fetal $(0.4 \pm 0.2$ and $0.6 \pm 0.3 \mu \mathrm{M}$, respectively) (means \pm SD) venous return. These concentrations representing carnitine release from placental tissue were much lower than those found in the venous returns during perfusion with carnitine or carnitine esters and were subtracted from the latter.

In four experiments where clearance, clearance index, and transfer could be evaluated for all carnitines, these parameters were slightly but not significantly higher for free carnitine than for acetylcarnitine (Table 1). The clearance and clearance index for D-glucose were clearly higher $(p<0.01$, Wilcoxon signed ranks test). No transfer of L-palmitoylcarnitine across the placenta could be detected.

If the maternal perfusate (maternal artery) contained only Lcarnitine, up to $30 \%$ of the total carnitine recovered at the maternal venous side consisted of esterified carnitine. If the maternal arterial perfusion medium contained only L-acetylcarnitine or L-palmitoylcarnitine, up to $10 \%$ of the total carnitine at the maternal venous side was in the free form. This was not due to spontaneous hydrolization of carnitine esters in the medium, since the carnitine esters used were shown to be stable under these conditions for at least $3 \mathrm{~h}$.

During perfusion with L-palmitoylcarnitine, up to $40 \%$ of the amount offered to the placental lobe was taken up by the placental tissue without being released into the fetal circulation.

Series II. In six of nine experiments a fetal to maternal concentration ratio (FV/MA) greater than 1 was seen for ${ }^{3} \mathrm{H}-\mathrm{L}$ lysine after recirculation of the fetal perfusate (Fig. 1). No such gradient could be demonstrated for L-carnitine or L-acetylcarnitine.

When the maternal arterial perfusion medium contained Lcarnitine, carnitine esters accumulated in the fetal circulation (Fig. 2a); whereas perfusion with L-acetylcarnitine resulted in the accumulation of free carnitine on the fetal side (Fig. $2 b$ ). No long-chain acylcarnitine esters could be detected in the fetal circulation.

Series III. The rate of appearance of ${ }^{14} \mathrm{C}$-L-carnitine (exchange rate) on the fetal side during fetal recirculation was about $40 \%$ that of ${ }^{3} \mathrm{H}$-L-lysine (Fig. 3). During the initial phase of the experiment, the rate of transfer of antipyrine was greater than that of ${ }^{3} \mathrm{H}$-L-lysine $(p<0.02)$. However, after $150 \mathrm{~min}$ of recirculation, the fetal concentration of ${ }^{3} \mathrm{H}$-L-lysine was greater than that of antipyrine ( $p<0.02$, Wilcoxon signed ranks test). While the concentration curve for lysine was still rising at 150 min, that for antipyrine was at a plateau. The maximal concentration of antipyrine in the fetal circulation remained below that in the maternal perfusate; whereas that of ${ }^{3} \mathrm{H}$-L-lysine exceeded the concentration in the maternal perfusate after $150 \mathrm{~min}$ of fetal recirculation in two experiments.

In all four experiments with differentially labeled L- and Disomers of carnitine, there was a larger uptake of ${ }^{14} \mathrm{C}$-L-carnitine from the maternal perfusate, but a higher exchange rate of ${ }^{3} \mathrm{H}$ D-carnitine across the placenta (Fig. 4, Table 2). Therefore, the relative placental uptake of $L-$ and D-isomers of carnitine (L:D) was always greater than 1 ; whereas the relative transplacental exchange was always below 1 (Table 2).

Placental retention of ${ }^{14} \mathrm{C}$-L-carnitine was higher than that of ${ }^{3} \mathrm{H}$-D-carnitine in most cases; the concentration of ${ }^{14} \mathrm{C}$-isotope in placental tissue exceeded that in the maternal perfusate (Tables

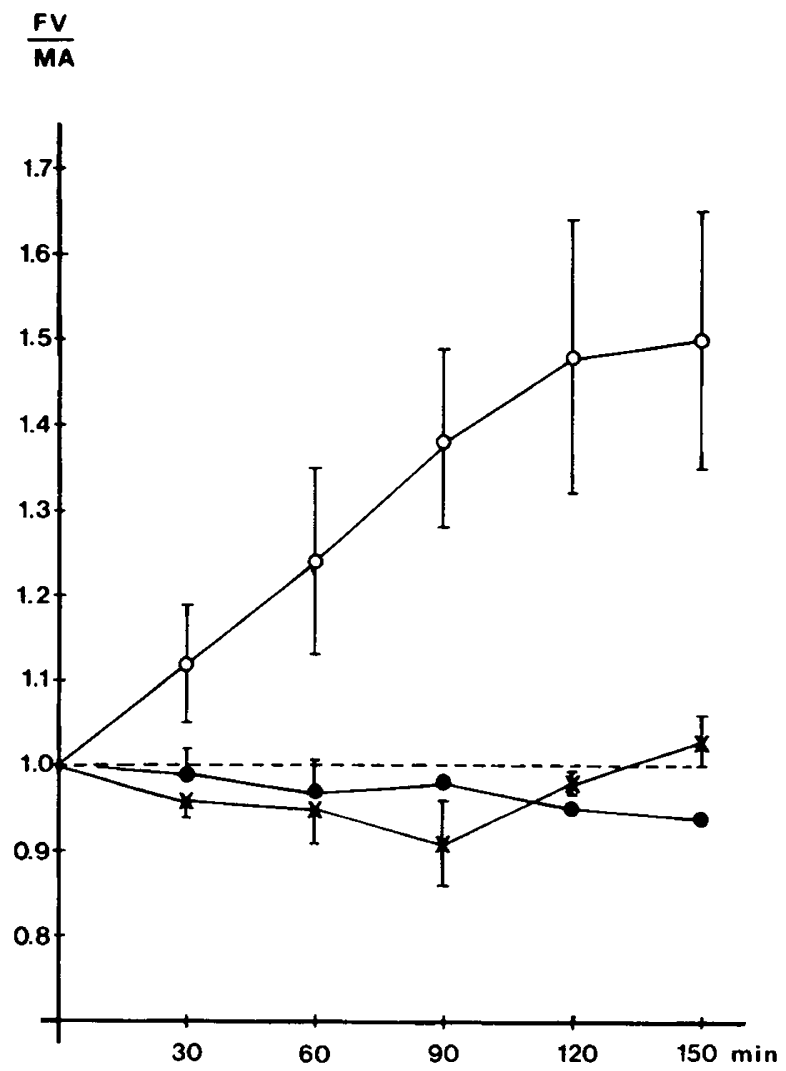

Fig. 1. Fetal $(F V) /$ maternal $(M A)$ concentration gradients of L-carnitine $(\bullet, n=3)$, L-acetylcarnitine $(x, n=3)$, and ${ }^{3} \mathrm{H}$-L-lysine $(\mathrm{O}, n=6)$ in relationship to the duration of fetal recirculation (mean \pm SD). Initially, the concentrations of all substances were equal on both sides of the placenta (FV/MA gradient $=1.0$ ). The maternal circulation remained open.

Table 1. Clearance, clearance index, and maximal transfer of L-carnitine, L-carnitine esters, D-glucose, and antipyrine across the in vitro perfused human placenta (means $\pm S D)^{*}$

\begin{tabular}{llccc}
\hline & $n$ & $\begin{array}{c}\text { Clearance } \\
\text { (ml/min) }\end{array}$ & $\begin{array}{c}\text { Clearance } \\
\text { index (\%) }\end{array}$ & $\begin{array}{c}\text { Transfer } \\
\text { (nmol/min.g placenta) }\end{array}$ \\
\hline L-Carnitine & 5 & $0.57 \pm 0.26$ & $20 \pm 6$ & $0.8 \pm 0.2$ \\
L-Acetylcarnitine & 4 & $0.41 \pm 0.11$ & $15 \pm 4$ & $0.7 \pm 0.3$ \\
L-Palmitoylcarnitine & 5 & 0 & 0 & 0 \\
D-Glucose & 5 & $1.87 \pm 0.52$ & $63 \pm 11$ & 100 \\
Antipyrine & 5 & $2.93 \pm 0.54$ & 10 & \\
\hline
\end{tabular}

\footnotetext{
* Both maternal and fetal circulations were kept open; see text for details.
} 

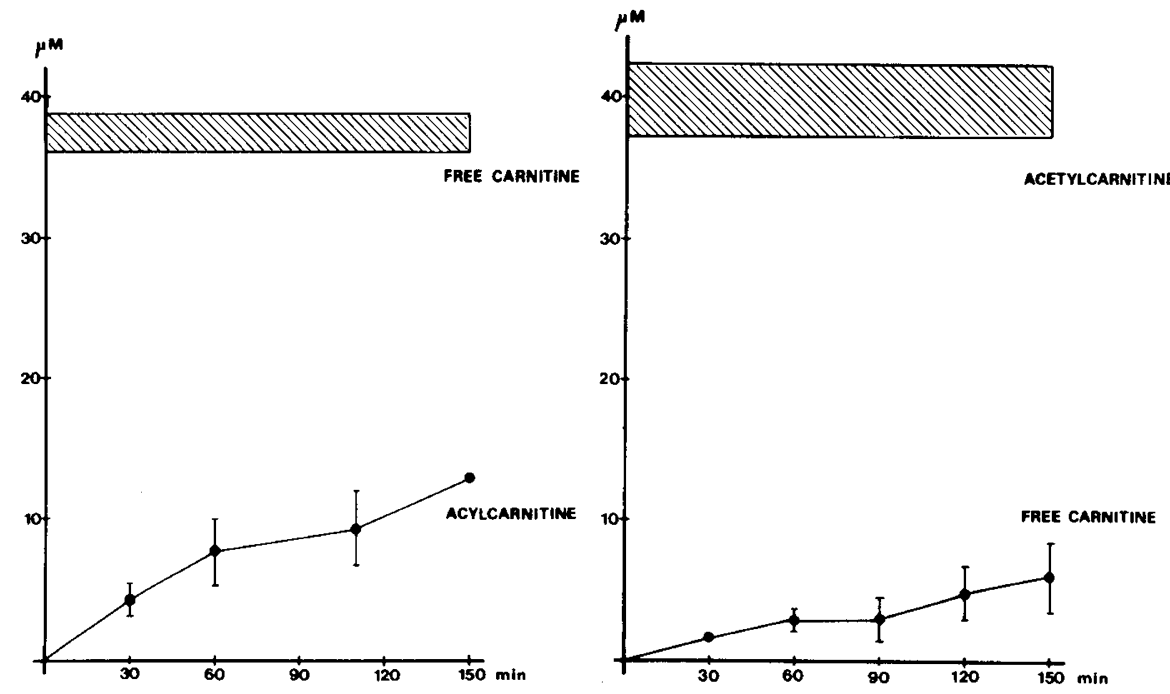

Fig. 2. Concentration of acylcarnitine (left panel, $n=5$ ) or free carnitine (right panel, $n=4$ ) in the fetal perfusate in relationship to the duration of fetal recirculation (mean \pm S.D.). At the beginning of the experiments, maternal and fetal perfusion media contained L-carnitine (right panel) or L-acetylcarnitine (left panel) in equal concentrations. The mean concentrations of free carnitine or acetylcarnitine present in the fetal perfusate are indicated by the shaded bars. The maternal circulation remained open.

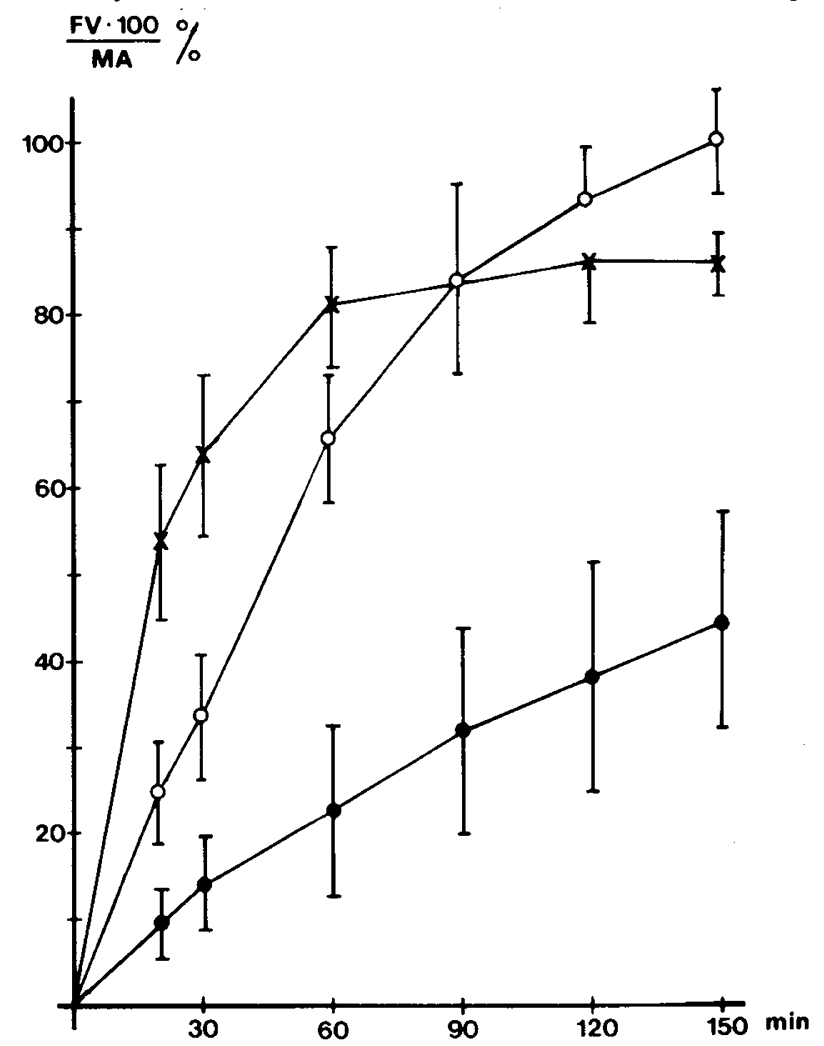

Fig. 3. Concentrations of ${ }^{14} \mathrm{C}$-L-carnitine $(\bullet, n=8),{ }^{3} \mathrm{H}$-L-lysine $(\mathrm{O}$, $n=4)$, and antipyrine (x, $n=4)$ in the fetal circulation expressed as percentage of the maternal arterial perfusate concentration (mean \pm SD). The $\mathrm{x}$-axis represents the duration of fetal recirculation. The concentrations of unlabeled L-carnitine and L-lysine were equal on both sides of the placenta. Radioactive tracers and antipyrine were only added to the maternal circulation which was kept open.

2 and 3). The relative placental retention (L:D) was always greater than 1 (Table 2). However, the specific activity of ${ }^{14} \mathrm{C}$-L-carnitine in the placenta remained far below that in the maternal perfusate (data not shown).

Chromatographic analysis of perfused placental tissue and recirculated fetal medium revealed that up to $40 \%$ of the total acid soluble ${ }^{14} \mathrm{C}$-radioactivity in the placenta and up to $6.5 \%$ of

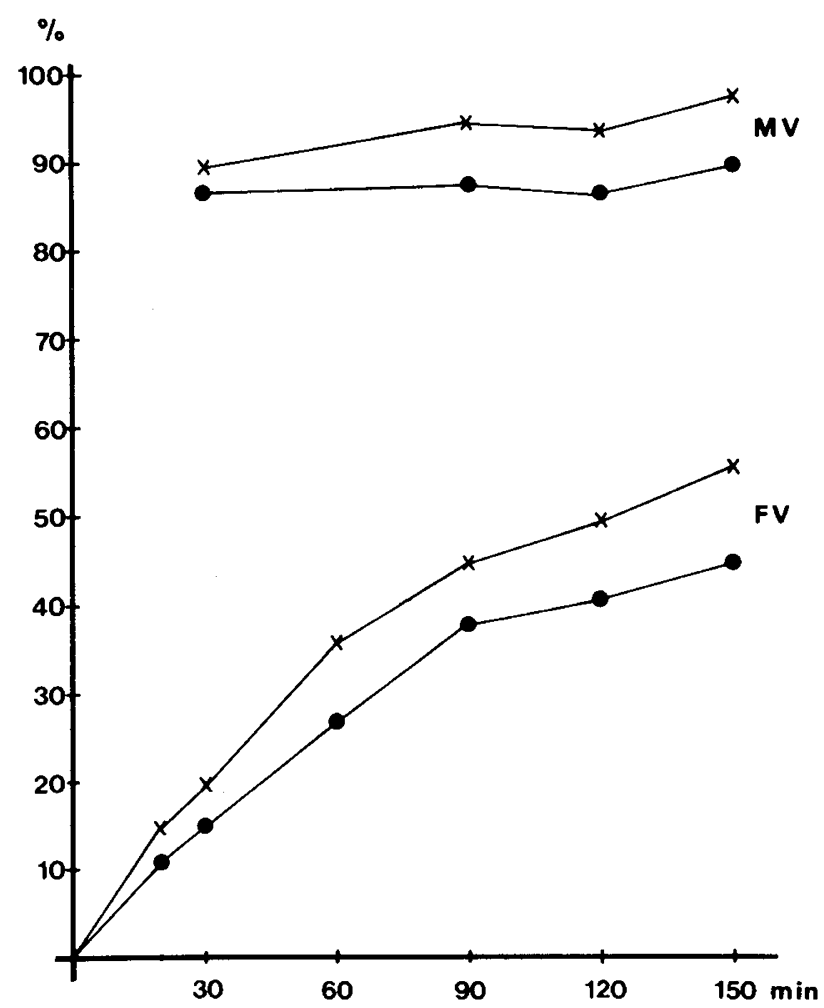

Fig. 4. Concentrations of ${ }^{14} \mathrm{C}$-L-carnitine $(\bullet)$ and ${ }^{3} \mathrm{H}$-D-carnitine (x) in the fetal circulation $(F V)$ and in the maternal venous perfusate $(M V)$, expressed as percentage of the concentrations in the maternal arterial perfusate. The values are related to the duration of fetal recirculation. The results of one representative experiment are given. The concentration of unlabeled D,L-carnitine was equal on both sides of the placenta. Radioactive tracers were only added to the maternal circulation which was kept open.

that in the fetal perfusate was recovered as acylated carnitine (Table 3). Between 1 and $2 \%$ of the total radioactivity in the placenta was recovered as long-chain acylcarnitine, but no radiolabeled long-chain acylcarnitine was detected in the fetal perfusate.

In seven unperfused placentas, the free carnitine concentration was about 15 times greater than that in maternal or umbilical 
Table 2. Placental uptake, transplacental exchange, and placental retention for ${ }^{14} C-L$ - and ${ }^{3} H$-D-carnitine in four experiments*

\begin{tabular}{|c|c|c|c|c|c|c|c|c|c|}
\hline \multirow[b]{2}{*}{ Experiment } & \multicolumn{3}{|c|}{$\begin{array}{l}\text { Placental uptake (\%) } \\
\frac{\text { MA - MV }}{\text { MA }} \cdot 100\end{array}$} & \multicolumn{3}{|c|}{$\begin{array}{l}\text { Transplacental exchange (\%) } \\
\frac{\mathrm{FV}}{\mathrm{MA}} \cdot 100\end{array}$} & \multicolumn{3}{|c|}{$\begin{array}{l}\text { Placental retention }(\%) \\
\frac{\mathrm{P}}{\mathrm{MA}} \cdot 100\end{array}$} \\
\hline & ${ }^{14} \mathrm{C}-\mathrm{L}$ & ${ }^{3} \mathrm{H}-\mathrm{D}$ & L:D & ${ }^{14} \mathrm{C}-\mathrm{L}$ & ${ }^{3} \mathrm{H}-\mathrm{D}$ & L:D & ${ }^{14} \mathrm{C}-\mathrm{L}$ & ${ }^{3} \mathrm{H}-\mathrm{D}$ & $L: D$ \\
\hline 1 & 6.7 & 5.0 & 1.34 & 32.6 & 39.1 & 0.83 & 70.0 & 54.6 & 1.28 \\
\hline 2 & 10.4 & 8.9 & 1.17 & 50.3 & 58.2 & 0.86 & 120 & 80.9 & 1.49 \\
\hline 3 & 12.9 & 10.5 & 1.23 & 26.6 & 35.9 & 0.74 & 110 & 92.9 & 1.18 \\
\hline 4 & 10.8 & 9.5 & 1.14 & 33.8 & 49.0 & 0.69 & 215 & 86.7 & 2.48 \\
\hline Mean & 10.2 & 8.5 & 1.22 & 35.8 & 45.6 & 0.78 & 129 & 78.8 & 1.61 \\
\hline
\end{tabular}

${ }^{*}{ }^{14} \mathrm{C}$-L- and ${ }^{3} \mathrm{H}$-D-carnitine were added to the maternal perfusate only. The maternal circulation was kept open and the fetal perfusate was recirculated. The isotope concentrations are expressed as percentage of the maternal arterial perfusate concentration (MA in cpm/ml), according to the formulas given herein, and are derived from samples taken after $60 \mathrm{~min}$ of fetal recirculation from the maternal venous (MV in cpm/ml) and fetal venous (FV in $\mathrm{cpm} / \mathrm{ml}$ ) perfusates or at the end of the experiments from perfused placental tissue (P in cpm/g wet weight). L:D are the respective isotope ratios (see formulas in "Materials and methods").

Table 3. Concentrations of total acid soluble ${ }^{14} \mathrm{C}$-radioactivity (TC) and fractions of ${ }^{14} \mathrm{C}$-labeled metabolites of carnitine in placental tissue and fetal perfusate at the end of fetal recirculation in five experiments*

\begin{tabular}{|c|c|c|c|c|c|c|}
\hline & \multicolumn{3}{|c|}{ Placental tissue } & \multicolumn{3}{|c|}{ Fetal perfusate } \\
\hline & $\begin{array}{c}\text { TC } \\
(\% \text { MA) }\end{array}$ & $\begin{array}{c}\mathrm{AcC} \\
(\% \text { TC) }\end{array}$ & $\begin{array}{l}\text { SCAC + } \\
\text { MCAC } \\
\text { (\% TC) }\end{array}$ & $\begin{array}{c}\mathrm{TC} \\
(\% \mathrm{MA})\end{array}$ & $\begin{array}{c}\mathrm{AcC} \\
(\% \mathrm{TC})\end{array}$ & $\begin{array}{l}\text { SCAC + } \\
\text { MCAC } \\
(\% \text { TC) }\end{array}$ \\
\hline 1 & 70 & 35.5 & 1.7 & 54.7 & 3.5 & 1.1 \\
\hline 2 & 120 & 28.5 & 4.0 & 80.7 & 4.9 & 1.5 \\
\hline 3 & 110 & 38.5 & 2.2 & 49.0 & 1.3 & $\dagger$ \\
\hline 4 & 215 & 28.7 & 7.4 & 65.1 & 1.0 & 4.2 \\
\hline 5 & 207 & 34.3 & 1.7 & 60.9 & 1.2 & 1.2 \\
\hline Mean & 144 & 33.1 & 3.4 & 62.1 & 2.4 & 2.0 \\
\hline
\end{tabular}

* The isotope concentrations of total soluble carnitine (TC) are expressed as percentage of the maternal arterial perfusate (MA) concentration. The isotope concentrations of acetylcarnitine (AcC) and short- + medium-chain acylcarnitines $(\mathrm{SCAC}+\mathrm{MCAC})$ are expressed as percentage of the ${ }^{14} \mathrm{C}$-labeled TC.

$\uparrow$ Not measurable.

blood (Table 4); $24 \%$ of the total acid soluble carnitine in placental tissue was in the esterified form.

\section{DISCUSSION}

The double circuit in vitro perfusion of an isolated lobe of human term placenta has been successfully used to study placental transfer of important substrates for fetal metabolism.

It has been established that in this system, ultrastructural and metabolic integrity of the placenta is maintained for up to $12 \mathrm{~h}$ (25). For amino acids and glucose, a stereo-specific carriermediated mechanism could be demonstrated which accelerates transfer of the natural isomer from the maternal to the fetal circulation $(16,18)$. Furthermore, the active transport of L-amino acids against a concentration gradient could be shown. It, therefore, seemed appropriate to apply this experimental approach to the study of the transplacental movement of carnitine.

As expected from measurements in maternal and umbilical blood, there is significant transfer of carnitine across the human term placenta. Our results do not exclude a different placental permeability to carnitine earlier in gestation. Although calculations based on in vitro transfer rates may not necessarily be appropriate for the estimation of in vivo transfer, they may give a rough indication of whether or not carnitine transfer across the placenta covers fetal requirements. Calculated on the basis of an average placental weight of $450 \mathrm{~g}$, the maximal transfer of carnitine from the maternal to the fetal circulation is about 500
Table 4. Concentrations (mean $\pm S D / g$ wet $w t$ ) of free and acid soluble acylcarnitine in seven unperfused human placentas compared with mean maternal venous and umbilical arterial and venous plasma levels

\begin{tabular}{lll}
\hline & \multicolumn{1}{c}{ Free carnitine } & \multicolumn{1}{c}{ Acylcarnitine } \\
\hline $\begin{array}{l}\text { Placental tissue } \\
\text { Plasma* } \\
\begin{array}{c}\text { Maternal ve- } \\
\text { nous }\end{array}\end{array}$ & $0.185 \pm 0.061 \mu \mathrm{mol} / \mathrm{g}$ & $0.057 \pm 0.022 \mu \mathrm{mol} / \mathrm{g}$ \\
$\begin{array}{c}\text { Umbilical ve- } \\
\text { nous }\end{array}$ & $0.010 \mu \mathrm{mol} / \mathrm{ml}$ & $0.010 \mu \mathrm{mol} / \mathrm{ml}$ \\
$\begin{array}{c}\text { Umbilical arte- } \\
\text { rial }\end{array}$ & $0.013 \mu \mathrm{mol} / \mathrm{ml}$ & $0.008 \mu \mathrm{mol} / \mathrm{ml}$ \\
\hline
\end{tabular}

${ }^{*}$ From Schmidt-Sommerfeld et al. (10).

$\mu \mathrm{mol} / \mathrm{day}$. The rate of accumulation of carnitine in the fetus during the last trimester of pregnancy was calculated to be 13 $\mu \mathrm{mol} / \mathrm{day}$, based on recent measurements of tissue carnitine concentrations at autopsy (25). Thus, the capacity of the placenta to deliver carnitine to the fetal circulation is far in excess of the estimated fetal carnitine requirement. Therefore, the accumulation of carnitine in fetal tissues would theoretically not require fetal biosynthesis.

Carnitine is transferred across the placenta in the form of free carnitine, acetylcarnitine [presumably the major fraction of carnitine esters in plasma (27)], and possibly other short-chain acylcarnitines. No transfer of palmitoylcarnitine [present in plasma in small amounts (28)] could be detected. This may be partly due to the strong binding of palmitoylcarnitine to albumin (Schmidt-Sommerfeld E, unpublished data), and possibly also to placental tissue protein, suggested by the large placental uptake of palmitoylcarnitine during perfusion. Protein binding is known to affect placental transfer of lipophilic compounds (29). There was also no measurable release of labeled long-chain acylcarnitines from the placenta into the perfusates. These findings suggest that long-chain fatty acids are not transferred across the placental membrane in the form of carnitine esters.

The mechanism of carnitine transfer from the maternal to the fetal circulation was investigated in series II and III. Although in series II, an uphill maternal-fetal concentration gradient could be established for the reference substance L-lysine, no such gradient was found for L-carnitine or L-acetylcarnitine (Fig. 1). This suggests that, in contrast to certain amino acids, there is no active transport of carnitine from the maternal to the fetal side of the placenta. In series III, the transplacental exchange rate of L-carnitine, an approximation of the maximal transfer rate (18), did not exceed that of the unphysiological D-isomer (Fig. 4).

As suggested from measurements of the specific activity of ${ }^{14} \mathrm{C}$ L-carnitine in placental tissue homogenate, full equilibration of 
the isotope with the intracellular L-carnitine pool was not achieved, probably due to the limited duration of the experiments. Whether this lack of equilibration affected transplacental exchange of isotope remains unclear. Therefore, a stereospecific transport system, accelerating the transfer of carnitine from the maternal to the fetal circulation, cannot be ruled out by these findings. However, similar experiments with differentially labeled L- and D-amino acids had shown a preferential transfer of the Lform even before an isotopic steady-state was achieved (18). We, therefore, consider the existence of such a transport system for L-carnitine to be unlikely. Furthermore, the clearance index which was measured for unlabeled L-carnitine (Table 1) is quite similar to that previously obtained for other hydrophilic compounds of comparable molecular size like D-leucine (18) or Lglucose (16), for which no transport system exists. It is well established that in the hemochorial placenta, hydrophilic molecules up to the size of inulin (molecular weight 5000) do not encounter steric hindrance during diffusion through large waterfilled channels in the extracellular space (30). The in vitro measured transfer of carnitine across the placental barrier is, therefore, compatible with passive diffusion.

However, placental uptake of ${ }^{14} \mathrm{C}$-L-carnitine was higher than that of ${ }^{3} \mathrm{H}$-D-carnitine and the concentration of ${ }^{14} \mathrm{C}$-isotope in the tissue extract was usually higher than that in the perfusion media (Table 2). This may reflect a stereospecific uptake of $L-$ carnitine and/or cellular sequestration of the label as carnitine ester. A cellular uphill transport mechanism of carnitine has not yet been described in the placenta, but is known to exist in a number of other tissues, e.g. muscle (31), heart (32), liver (33), and kidney (34).

Our results also suggest that the human term placenta plays an active role in carnitine metabolism since perfusion with $\mathrm{L}$ carnitine resulted in the release of short-chain acylcarnitines into the fetal, as well as maternal, circulation. On the other hand, free carnitine was detected in both perfusates when the placental lobe was perfused with L-acetylcarnitine. A strikingly high placental uptake of L-palmitoylcarnitine was observed.

The recovery of radiolabeled carnitine esters from placental tissue following perfusion with ${ }^{14} \mathrm{C}$-L-carnitine proved that free carnitine of maternal origin can be esterified by the placenta to short- and long-chain acylcarnitine esters. In agreement with the literature (13), the concentration of acid soluble acylcarnitine in unperfused placenta was $24 \%$ of total acid soluble carnitine (Table 4). An even higher percentage was recovered in perfused placental tissue at the end of our experiments (Table 3 ).

Our findings are compatible with earlier investigations that demonstrated the presence of carnitine acyltransferase and carnitine palmitoyltransferase activities in the human placenta (1214), as well as the capacity of human placental tissue to oxidize ${ }^{14} \mathrm{C}$-palmitate in vitro (35), and of placental mitochondria to oxidize palmitoylcarnitine (36).

Little is known about the physiological significance of fat catabolism in the placenta. It has been suggested that fatty acids are not the principal energy yielding substrates for the human placenta (37). However, the relative utilization of glucose versus fatty acids by the placenta under different metabolic conditions, e.g. maternal diabetes mellitus or toxemia of pregnancy, has not yet been investigated. The significance of placental fatty acid oxidation for substrate transfer to the fetus is also not known. The limited release of carnitine esters into the fetal circulation suggests that under normal metabolic conditions, placental formation of acylcarnitine through $\beta$-oxidation may not be quantitatively important for the supply of high energy substrates to the fetus. The formation of acetylcarnitine may instead reflect the placenta's ability to reduce a potentially toxic accumulation of intramitochondrial acetyl coenzyme $A$ and to maintain the intramitochondrial free coenzyme A pool. Such a mechanism for reducing intramitochondrial acetyl coenzyme A "pressure" has been suggested for other tissues (38).

In summary, at least three mechanisms seem to be involved in placental carnitine transport: 1) passive diffusion through extracellular channels; 2) stereospecific cellular uptake; and 3) intracellular modification and release of metabolites. We suggest that mechanism one is quantitatively the most important for the supply of carnitine to the fetus, while mechanisms two and three play roles in placental tissue metabolism.

Acknowledgments. The authors thank Mrs. M. Lützen and Mrs. B. Benz for their technical assistance.

\section{REFERENCES}

1. Fritz IR, Marquis NR 1965 The role of carnitine esters and carnitine palmitoyltransferase in the transport of fatty acyl groups across mitochondrial membranes. Proc Natl Acad Sci USA 54:1226-1232

2. Schmidt-Sommerfeld E, Novak M, Penn D, Wieser PB, Buch M, Hahn P 1978 Carnitine and the development of newborn adipose tissue. Pediatr Res 12:660-664

3. Wolf H, Stave U, Novak M, Monkus EF 1974 Recent investigations on neonatal fat metabolism. J Perinat Med 2:75-87

4. Schiff D, Chan G, Seccombe D, Hahn P 1979 Plasma carnitine levels during intravenous feeding of the neonate. J Pediatr 95:1043-1046

5. Penn D, Schmidt-Sommerfeld E, Wolf H 1980 Carnitine deficiency in premature infants receiving total parenteral nutrition. Early Hum Dev 4:23-34

6. Novak M, Monkus EF, Chung D, Buch M 1981 Carnitine in the perinatal metabolsm of lipids. I. Relationship between maternal and fetal plasma levels of carnitine and acylcarnitines. Pediatrics 67:95-100

7. Penn D, Schmidt-Sommerfeld E., Pascu F 1981 Decreased tissue carnitine levels in newborn infants receiving total parenteral nutrition. J Pediatr 98:976-78

8. Rebouche CJ 1980 Comparative aspects of carnitine biosynthesis in microorganisms and mammals with attention to carnitine synthesis in man. In Frenkel RA, McGarry JD (eds) Carnitine Biosynthesis, Metabolism and Functions. Academic Press, New York, pp 57-72

9. Hahn P, Seccombe DW, Towell ME 1981 The perinatal role of carnitine. In: Monset-Couchard M, Minkowski A (eds) Physiological and Biochemical Basis for Perinatal Medicine. S. Karger, Basel, pp 187-198

10. Schmidt-Sommerfeld E, Penn D, Wolf $H$ 1981 The influence of maternal fat metabolism on fetal carnitine levels. Early Hum Dev 5:233-242

11. Hahn P, Skala JP, Seccombe DW, Frohlich, J, Penn-Walker D, Novak M, Hynie I, Towell ME 1977 Carnitine content of blood and amniotic fluid. Pediatr Res 1 1:878-880

12. Hahn P, Skala J 1973 Carnitine transferases in human fetal tissues. Biol Neonate 22:9-15

13. Welsch F, Wenger WC 1980 Carnitine and carnitine acetyltransferase in the placenta of mouse, marmoset and man. Comp Biochem Physiol 67B:97101

14. Karp W, Sprecher H, Robertson, A 1971 Carnitine palmitoyltransferase activity in the human placenta. Biol Neonate 18:341-347

15. Schneider H, Panigel M, Dancis J 1972 Transfer across the perfused human placenta of antipyrine, sodium and leucine. Am J Obstet Gynecol 114:822 828

16. Schneider H, Challier J-C, Dancis J 1981 Transfer and metabolism of glucose and lactate in the human placenta. In: Young M, Boyd RDH, Longo LD, Telegdy G (eds) Placental Transfer: Methods and Interpretations. WB Saunders, London, pp 129-137.

17. Dancis J, Jansen V, Kayden HJ, Schneider H, Levitz M 1973 Transfer across the perfused human placenta. II. Free fatty acids. Pediatr Res 7:192-197

18. Schneider H, Möhlen K-H, Dancis J 1979 Transfer of amino acids across the in vitro perfused human placenta. Pediatr Res 13:236 -240

19. McGarry JD, Foster DW 1976 An improved and simplifed radioisotopic assay for the determination of free and esterified carnitine. J Lipid Res 17:277281

20. Brass EP, Hoppel CL 1978 Carnitine metabolism in the fasting rat. J Biol Chem 253:2688-2693

21. Huggett ASG, Nixon DA 1957 Use of glucose oxidase peroxidase and Odianisidine in determination of blood and urinary glucose. Lancet 2:368 370

22. Czok R, Lamprecht W 1974 Pyruvate, phosphoenolpyruvate and D-glycerate2-phosphate. In: Bergmeyer HU (ed) Methods of Enzymatic Analysis. p 1446 (Academic Press, Inc, New York, pp 1446-1449

23. Noll F L-(+)-Lactate. Determination with LDH, GPT and NAD. In: Bergmeyer U (ed) Methods of Enzymatic Analysis. Academic Press, Inc, New York, pp $1475-1477$

24. Brodie BB, Axelbrod J, Soberman R, Levy BB 1949 The estimation of antipyrine in biological materials. J Biol Chem 179:25-29

25. Miller RK, Wier PJ, Maulik D, Di Sant'agnese PA 1985 Human placenta in vitro: characterization during 12 hours of dual perfusion. In: Schneider $\mathrm{H}$ Dancis J (eds) In Vitro Perfusion of Human Placental Tissue. Internationa Workshop Zürich, 1984. Contr Gynecol Obstet 13:141-143, Karger, Basel

26. Penn D, Ludwigs B, Schmidt-Sommerfeld E, Pascu F 1985 Effect of nutrition on tissue carnitine concentrations in infants of different gestational ages. Biol Neonate 47:130-135

27. Valkner KJ, Bieber LL 1982 Short-chain acylcarnitines of human blood and urine. Biochem Med 28:197-199 
28. Schmidt-Sommerfeld, E Penn D, Wolf H 1983 Carnitine deficiency in premature infants receiving total parenteral nutrition: effect of L-carnitine supplementation. J Pediatr 102:931-935

29. Dancis J, Jansen V, Levitz M 1976 Transfer across perfused human placenta. IV. Effect of protein binding on free fatty acids. Pediatr Res 10:5-10

30. Faber JJ, Thornburg KL 1981 The forces that drive inert solutes and water across the epitheliochorial placentae of the sheep and the goat and the hemochorial placentae of the rabbit and the guinea pig. In: Young M (ed). Placental Transfer: Methods and Interpretations. Placenta Suppl. 2, pp 203-213

31. Rebouche CJ 1977 Carnitine movement across muscle cell membranes. Studies in isolated rat muscle. Biophys Acta 471:145-155

32. Vary TC, Neely JR 1982 Characterization of carnitine transport in isolated perfused adult rat hearts. Am J Physiol 242:H585-H592

33. Christiansen RZ, Bremer J 1976 Active transport of butyrobetaine and carni- tine into isolated liver cells. Biochim Biophys Acta 448:562-577

34. Huth PJ, Shug AL 1980 Properties of carnitine transport in rat kidney cortex slices. Biochim Biophys Acta 602:621-634

35. Yoshioka T, Roux JF 1972 In vitro metabolism of palmitic acid in human fetal tissue. Pediatr Res 6:675-81

36. Swierczński J, Scisowski A, Aleksandrowicz Z 1976 Oxidation of palmitoylcarnitine by mitochondria isolated from human term placenta. Biochem Med 16:55-58

37. Beaconsfield $P$ Ginsburg J 1979 Carbohydrate, fat and protein metabolism in the placenta: A clinician's review. In: Beaconsfield P, Villee C (eds) Placenta-A Neglected Experimental Animal. Pergamon Press, Oxford, pp 34125

38. Bieber LL, Emaus R, Valkner K, Farrell S 1982 Possible functions of shortchain and medium-chain carnitine acyltransferases. Fed Proc 41:2858-2862

\title{
Aminophylline Reduces Hypoxic Ventilatory Depression: Possible Role of Adenosine
}

\author{
ROBERT A. DARNALL, JR. \\ Department of Pediatrics, University of Virginia Medical Center, Charlottesville, Virginia 22908
}

\begin{abstract}
Newborn infants and animals typically exhibit a paradoxical ventilatory response to hypoxia. The depressive phase of the response has not been adequately explained. It has been suggested that hypoxia may cause the release of inhibitory neuromodulators which depress ventilation. We have postulated that the nucleoside, adenosine, may be involved because 1 ) it is rapidly released during hypoxia, 2) it depresses ventilation, and 3) theophylline, a competitive inhibitor, has successfully been used to treat apnea of prematurity. Herein we describe the effects of aminophylline on ventilation during hypoxia in the spontaneously breathing newborn piglet administered both rapidly after ventilatory depression has occurred (bolus) and before the onset of hypoxia (pretreatment). Ten percent oxygen breathing produced a typical biphasic ventilatory response. The decrease in minute ventilation was caused by a decrease in both tidal volume and respiratory frequency. The bolus administration of aminophylline reversed the depression in minute ventilation $(p<$ $0.001)$ by increasing tital volume $(p<0.002)$. Pretreatment with aminophylline decreased the amount of ventilatory depression $(p<0.05)$ by preventing a decrease in respiratory frequency. We conclude that aminophylline, an adenosine antagonist, reduces the decrease in ventilation which occurs during hypoxia in the newborn. We speculate that adenosine may play a role in hypoxic ventilatory depression and respiratory control in the newborn. (Pediatr Res 19: 706-710, 1985)
\end{abstract}

\section{Ve, minute ventilation \\ f, respiratory frequency}

Abbreviations

Received April 16, 1984; accepted March 7, 1985.

Correspondence Dr. Robert A. Darnall, Jr., Department of Pediatrics, Box 386 , University of Virginia Medical Center, Charlottesville, VA 22908.

Supported by Biomedical Research Support Award S07-RR05431-20 and by a grant from the American Lung Association.

\author{
$V t$, tital volume \\ HR, heart rate \\ BPs, systolic blood pressure
}

The paradoxical ventilatory response to hypoxia has been well documented in the newborn animal and human, and may contribute to the etiology of neonatal apnea. The neonate typically exhibits a biphasic ventilatory response to hypoxia (1-3) characterized by a brief increase in Ve, followed by a sustained decrease which is further accentuated by the resumption of normoxic or hyperoxic gas inhalation. The initial increase in ventilation has conventionally been explained as reflecting peripheral chemoreceptor stimulation. The subsequent decrease, however, has not been satisfactorily explained. A failure of peripheral chemoreceptors is probably not involved (4-6) but a number of other mechanisms have been suggested including alterations in pulmonary mechanics (7), and hypoxic depression of central respiratory centers (8).

Central depression of respiration might be caused by a decrease in metabolic rate (9), alterations in cerebral blood flow (10), rapid exhaustion of facilitory neurotransmitter stores (11-13), or the release of rapid acting inhibitory neuromodulator such as endogenous opioids (14) or GABA (15). We have recently postulated that the nucleoside, adenosine, may also be a candidate as an inhibitory neuromodulator $(16,17)$. Our postulate is based on three lines of evidence: 1) theophylline, a specific competitive inhibitor of adenosine, is effective in treating neonatal apnea $(18) ; 2)$ adenosine is rapidly released in the brain during hypoxia (19); and 3) adenosine depresses ventilation when administered systemically or applied to the brainstem (20). Herein we describe the effects of theophylline on the hypoxic ventilatory response both when administered prior to the onset of hypoxia (pretreatment) and when given rapidly during hypoxia (bolus). 